\title{
Postures épistémologiques et cadres théoriques des principaux courants de l'éducation aux territoires
}

Yves Girault et Angela Barthes

\section{(2) OpenEdition \\ 12 Journals}

Édition électronique

URL : http://journals.openedition.org/ere/755

DOI : 10.4000/ere.755

ISSN : 2561-2271

Éditeur

Centr'ERE

Édition imprimée

Date de publication : 20 décembre 2016

ISSN : 1373-9689

\section{Référence électronique}

Yves Girault et Angela Barthes, «Postures épistémologiques et cadres théoriques des principaux courants de l'éducation aux territoires », Éducation relative à l'environnement [En ligne], Volume 13 - 2 | 2016, mis en ligne le 20 décembre 2016, consulté le 21 février 2020. URL : http:// journals.openedition.org/ere/755 ; DOI : 10.4000/ere.755 


\title{
Postures épistémologiques et cadres théoriques des principaux courants de l'éducation aux territoires
}

\author{
Yves Girault et Angela Barthes
}

1 En géographie et en sciences sociales en général, l'utilisation du terme de territoire émerge dans les années $1980^{1}$. Selon Bonnemaison (1981, p. 253) la première définition de ce terme a été proposée par Elliot Howard, ornithologue anglais, en se rapportant à la vie sociale des fauvettes. Elliot Howard (1920), cité dans Ardrey (1966, p. 39) proposa comme définition de la territorialité animale « la conduite caractéristique adoptée par un organisme pour prendre possession d'un territoire et le défendre contre les membres de sa propre espèce». Ce comportement, qualifié par Howard de " territorialité innée », fut repris par les éthologues qui l'ont étendu à d'autres sociétés animales. Par la suite, ce concept fut transposé dans le cadre des études des sociétés humaines par des chercheurs en sciences sociales pour lesquels le territoire était un «ensemble de lieux hiérarchisés, connectés à un réseau d'itinéraires, chargés culturellement et symboliquement» (Bonnemaison, 1981). Le terme territoire se complète et se précise ensuite avec l'ouvrage de Claude Raffestin (Raffestin, 1980) qui le spécifie non seulement comme lieu de vie quotidienne, mais également comme lieu de pouvoirs.

2 À partir des années 80 , on assiste à « une véritable inflation dans les usages du terme de territoire ", tant dans le nombre d'utilisateurs que dans les disciplines scientifiques qui en font l'usage (Rippol et Veschambre, 2002), et on note graduellement une évolution de l'échelle spatiale évoquée par le terme territoire : «d'échelle d'abord nationale [sa taille] est maintenant transposée à toutes les autres [échelles], avec une nette préférence pour l'échelle locale» (Di méo, 1998). Di Méo (1998), souligne "que le vocable [le territoire] s'étend aujourd'hui à d'innombrables domaines de la vie sociale, économique et culturelle ».

3 Par-delà les nombreux débats qui émanent aujourd'hui des différents champs disciplinaires des sciences humaines et sociales et de ? la géographie en particulier, une 
certaine conception commune de la notion de territoire semble dominer, soit «le rapport entre une société (entité globale) et une portion d'espace géographique " (Ripoll et Veschambre, 2002). Cette conception vient compléter les assertions dominantes des années 80 (le territoire comme lieu de vie et le territoire comme lieux de pouvoir) en précisant que le rapport entre une société et son espace, conçu comme un lieu de pouvoir, est aussi un "rapport d'appropriation» (Idem). Aujourd'hui, il semble que ce sont les acceptations du territoire comme "lieux de vie», "lieux de pouvoirs » et "rapports entre une société et son espace " (au sens sociologique du terme) qui sont le plus souvent reprises, dans des proportions différentes, dans le champ de l'éducation au territoire.

4 À partir de ces constats, nous souhaitons préciser comment s'insèrent en sciences de l'éducation les approches épistémologiques du territoire des géographes et comment celles-ci sont reprises dans les différents courants éducatifs jusqu'à l'émergence progressive du champ de l'éducation aux territoires.

\section{Approches épistémologiques du territoire de la communauté des géographes reprises dans les champs éducatifs : les courants des géographies sociales et culturelles et leurs prolongements}

5 La géographie sociale, un des courants les plus anciens de la géographie humaine (1870), doit beaucoup à Vidal de la Blache (1913), qui s'intéresse particulièrement aux relations entre les Hommes et leurs milieux. Après la deuxième Grande Guerre mondiale, Pierre George (1945) fonde "une géographie sociale du monde », qui est reprise dans les pays germanophones et anglophones (Hartke, 1969 ; Bobeck, 1969). Celle-ci s'intéresse plus précisément aux ségrégations spatiales. C'est dans les années 70 (Claval, 1973) puis 80 (Frémont et coll., 1984), que se structure la géographie sociale en tant que courant à part entière, s'intéressant alors aux sociétés dans leurs espaces et à leurs rapports de domination et d'appropriation. La géographie sociale postule que les faits de société constituent l'agent décisif de l'explication géographique. Tel que le conçoivent Guy Di Meo et Pascal Buleon (2005), « l'objet de la géographie sociale est l'étude des rapports existants entre rapports sociaux et rapports spatiaux ». L'objectif de la géographie sociale est de fonder une géographie de l'action, ouverte sur la société et sur les grandes questions sociales qui impliquent l'espace géographique.

6 La géographie culturelle est introduite par la géographie allemande (Butman et Ratzel, 1977) puis se développe en Amérique du Nord en s'intéressant particulièrement aux spécificités des différentes aires culturelles. La géographie culturelle apparait plus précisément en France dans les années 80 ; elle est formalisée par Paul Claval (1995, p. 101) qui définit « la culture comme l'ensemble des formes acquises de comportement et de connaissances dans une aire culturelle donnée ", et par Bonnemaison (2000, p. 86) qui précise que «le territoire peut être défini comme l'envers de l'espace. Il est idéel et même souvent idéal, alors que l'espace est matériel. Il est une vision du monde avant d'être une organisation; il ressort plus de la représentation que de la fonction, mais cela ne signifie pas qu'il soit pour autant démuni de structures et de réalité ». La géographie culturelle a donc mis en évidence l'importance des représentations, notamment des «imaginations géographiques", des savoirs, connaissances et 
comportements induits, des images du monde construites par les géographes et non géographes pour la compréhension du monde qui nous entoure (Houssay-Holzschuch, 2006).

$7 \quad$ En réalité de plus en plus d'auteurs comme Guy Di Méo proposent des définitions qui font la synthèse entre les approches culturelles et sociales. D'une part, elles intègrent les dimensions identitaires et politiques des territoires et d'autre part, elles introduisent l'idée de projets collectifs (sans que ce terme ne soit clairement discuté) : "le territoire est créé par l'appropriation (économique, idéologique, politique et sociale) d'un espace par des groupes ayant une représentation d'eux-mêmes et de leur histoire » (Di Méo, 1997, p. 43).

Cette synthèse et ses prolongements renvoient également aux rapports d'appropriation entre une société et son espace, et introduit aussi deux notions importantes qui viendront impacter les questions éducatives surtout en termes de finalités et valeurs : celle de l'identité du territoire et celle de ressource. La prise en compte de ces deux notions nécessite donc de ré-expliciter la notion de rapports d'appropriation de son espace par une société.

En effet, certains auteurs associent l'appropriation d'un espace au concept d'identité du territoire (Bretherton, 2001), soit la capacité des Hommes à co-construire et s'identifier à un ensemble de caractéristiques spécifiques d'un territoire. Mobilisées également par le courant de l'intelligence territoriale (Bertacchini, 2008), l'identité et l'appropriation sont alors liées. Dans ce cadre, Bertacchini $(2008$, p. 6) définit l'idée d'appropriation comme un "phénomène de représentation symbolique par lequel les groupes humains pensent leurs rapports à un espace matériel, ne [pouvant] se construire que par l'intégration progressive d'un sentiment local, au travers d'un processus mental collectif». C'est ce processus mental collectif d'adhésion à des valeurs qui forme l'identité d'un territoire, en tant qu'ensemble et qui permet d'effectuer le pont avec l'éducation. Les géographies sociales et surtout culturelles interpellent aussi la question des différents groupes sociaux et les différents registres de savoirs, lesquels doivent servir de base à la formation des identités territoriales. Leur émergence fait appel à ce que ces géographies nomment les «apprentissages collectifs». Il y a donc, dans les géographies sociales et culturelles, une dimension éducative valorisée qui se profile de facto en filigrane. Le territoire peut alors être considéré comme une réordination de l'espace informé, dans le sens ou l'espace contient une série d'informations lesquelles concourent à la structuration de l'existence pratique des individus dans ce territoire doté d'une identité cohérente.

10 Mais cet énoncé contient aussi une ambiguïté qui lie l'appropriation à la ressource. En effet, la construction identitaire des territoires peut aussi contenir, dans le contexte mondialisé et compétitif actuel, l'idée directe de son attractivité (les avantages comparés entre les lieux) voire de ses potentialités économiques. Ainsi, d'autres auteurs se référant aux rapports d'appropriation entre une société et son espace conçoivent le territoire comme «des ressources matérielles et symboliques ayant la capacité de structurer l'existence pratique des individus tout en étant créateur d'identité » Debarbieux (2009, p. 78). Là encore, certains géographes complètent cette réflexion en indiquant que cette ressource n'existe pas en tant que telle, mais nécessite d'être « révélée » pour reprendre les termes de Landel et Sénil (2009) par une série de savoirs mobilisés, lesquels concourent aussi à la structuration d'actions collectives. François et coll. (2008), à propos de la ressource, parlent «d'émergence et de 
reconnaissance partagée " pour laisser place à la notion de "ressource révélée " développée plus spécifiquement par Landel et Sénil (2009). Ainsi, à travers l'épistémologie de la géographie et ses prolongements récents, la notion d'appropriation (identité/ressource) donne lieu à diverses assertions qui vont, comme nous le montrerons, se décliner de façons très différentes dans les questions éducatives. Nous pouvons donc retenir que si la géographie définit les acceptions du territoire comme "lieux de vie», "lieux de pouvoirs» et "rapports entre une société et son espace ", les approches épistémologiques des courants de la géographie sociale et de la géographie culturelle introduisent aussi les liens entre les territoires et les savoirs. Elles formalisent ainsi l'importance de l'information dans les processus de coconstructions des savoirs qui induisent une construction territoriale (il est souvent fait référence à la création d'un espace informé). Elles formalisent également les contextes culturels (les représentations culturelles et les imaginaires géographiques) et s'intéressent alors aux processus de formation des identités (l'identité territoriale). Elles préfigurent enfin, à travers la notion complexe de rapport d'une société à son espace, la double finalité d'une appropriation territoriale, celle de la définition de l'identité des territoires et celle de l'exploitation des ressources territoriales. La figure 1 résume les fondements épistémologiques de la géographie par la suite utilisés dans l'éducation aux territoires.

L'ÉPISTÉMOLOGIE DE LA GÉOGRAPHIE ÉNONCE LES DIFFÉRENTES ACCEPTIONS DU TERRITOIRE

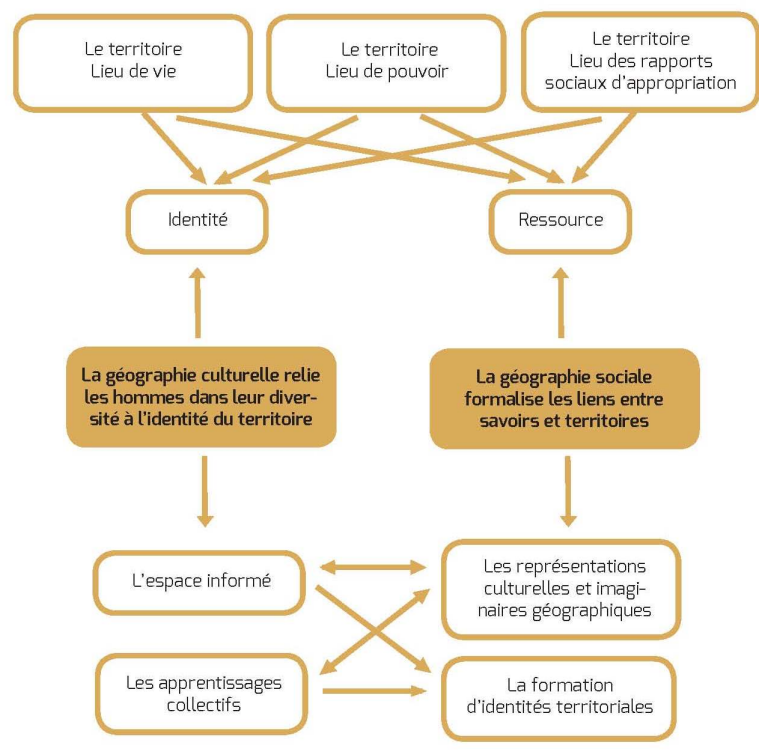

Figure 1: Les fondements épistémologiques de la géographie utilisés dans l'éducation à l'environnement en lien avec l'idée de territoires. 


\section{Les différents courants éducatifs en éducation relative à l'environnement centrés sur une éducation aux territoires}

\section{Courants d'éducation relative à l'environnement se référant au territoire en tant que « lieux de vie»}

12 Le premier auteur, nous semble-t-il, dont les travaux ont un lien avec ce que nous qualifions actuellement d'éducation aux territoires en tant que « lieux de vie », est John Muir (1838-1914), fondateur du Sierra Club et qualifié par de nombreux Américains de Father of our National Parks, Wilderness Prophet et de Citizen of the Universe. Ses travaux ont très largement influencé tout le courant de l'interprétation défini par Freeman Tilden comme « une activité éducative qui veut dévoiler la signification des choses et leurs relations par l'expérience personnelle et des exemples plutôt que par la seule communication de renseignements concrets " (1957, p. 119). L'interprétation s'est depuis très largement développée dans le cadre de la muséologie ${ }^{2}$. Parallèlement, et sous l'impulsion de Georges Henri Rivière, ont été créés des écomusées ${ }^{3}$ qui proposent un ancrage historique des habitants en rejoignant le courant nord-américain de l'interprétation où l'on fait appel à une expérience directe et concrète du lieu, souvent dans une approche historique, pour faire émerger chez les personnes un questionnement, une réflexion, un sentiment d'appartenance, etc. ${ }^{4}$

13 Par ailleurs, quatre courants d'éducation relative à l'environnement (ERE) sont intimement liés à la conception d'une éducation au territoire en tant que lieu de vie ${ }^{5}$. On trouve pro ou prou des prémisses de ceux-ci dans divers concepts qui décrivent une relation privilégiée avec l'environnement. Il s'agit notamment de «l'identité écologique » (Thomashow, 1995) qui fait référence à un lien d'attachement (Hay, 1988), de la topophilie (Tuan, 1991), de l'identité aux lieux (Proshansky, Fabian et Kaminoff, 1983), de l'identité communautaire (Hummon, 1986), d'intériorité (Relph, 1976) et de sensibilité environnementale (Tanner, 1980).

Dans ce cadre, Diane Pruneau et Olivier Chouinard (1997, 1998-1999) ont proposé un modèle d'intervention pédagogique qui favorise la relation personne / groupe social / environnement. Le territoire comme lieu de vie suscite un « attachement biorégional » qui, pour ces auteurs, « apparaît comme une étape préliminaire en éducation relative à l'environnement. Cette étape où l'apprenant accorde de la valeur à l'objet d'apprentissage (l'environnement) est nécessaire pour éventuellement le conduire à une implication environnementale et communautaire » (Pruneau, Gravel et Ouattara, 2002, p. 566).

15 Trois autres courants, l'écoformation (Pineau, 1983, 1992, 2001, Cottereau 2001), l'écoontogenèse (Berryman 2003), et « l'autobiographie environnementale (Berryman 2007, Bachelart 2009a, 2009b, Pineau 2012, Pineau et al 2013) valorisent également le territoire comme lieu de vie.

L'écoformation est un concept qui traduit tout ce champ relationnel par lequel « l'oikos » participe à la formation d'un être ou d'une personne, tout autant que l'être transforme son oikos (Pineau et coll., 1992, cité par Cottereau, 2000, p. 174) ${ }^{6}$. Dominique Cottereau s'appuie ainsi sur une pédagogie du sensible, de l'émotionnel et de l'imaginaire, qui, «semble devoir être introduite pour permettre une réelle rencontre 
avec l'environnement et l'établissement d'un lien "éco-logique " (de la logique de l'habitat) (Cottereau, 2000, p. 174). Comment peut donc s'inscrire la pédagogie de l'imaginaire, de l'écoformation, dans une éducation au territoire? Christophe Andreux (2005, p. 45) contribue à répondre à cette question en faisant référence à un projet lié au territoire montagnard qui a fait l'objet de trois années d'étude (Andreux et Braux, 2003). Selon Andreux (2005, p. 45), « la démarche pédagogique de l'alternance liant les pédagogies de projet et de l'écoformation permet de prendre en compte et de révéler la dimension de "territoire de vie partagée " (Andreux, 2001-2002)». Ainsi, cet auteur précise que tous les acteurs du projet (élus enseignants animateurs) sont «amenés à goûter, sentir toucher endurer, écouter, parler, réfléchir, créer, rêver, à s'émerveiller à vivre la montagne par tout leur corps et leur sens » (p. 4 6).

L'éco-ontogenèse, selon (Berryman, 2003, p. 209)7 , « est un processus s'étendant à la durée entière de la vie [...] qui fait référence à un phénomène la genèse de l'être [...] relié à l'environnement et engagé, tout au long de sa vie et de manière réflexive, dans ses relations ». Perazzone Camino et Bertolino (1999, p. 106) estimaient qu'une «très vaste littérature souligne l'importance d'un rapport intime et continuel des enfants avec la nature ». Ainsi, «l'enfant se lie [...] à l'environnement et apprend à s'y trouver ou s'y construire une place à lui. Il apprend à être bien dans le monde en dehors de sa famille et à y trouver une terre d'accueil. [...] En jouant dans la nature, l'enfant échange avec elle et apprend à se sentir bien dans le monde. [...] C'est concrètement qu'il se lie au monde dont il explore et manipule la structure» (Berryman, 2003, p. 218). Dans cette perspective, une éducation à l'environnement qui tient réellement compte du développement de l'enfant devrait lui permettre avant tout d'explorer et de se “ connecter" avec son propre lieu de vie. «Cette résonance du milieu dans la constitution de notre être n'agit pas qu'au seul niveau personnel, mais aussi comme partie prenante sociale, communautaire et culturelle, dans la culture matérielle et symbolique " (Berryman, 2005, p. 68).

18 Ainsi, à l'instar de Bonnemaison $(1996$, p. 167) pour qui la territorialité humaine est avant tout définie comme « la relation culturelle [qu'un groupe] entretient avec [...] le maillage des lieux et le système d'itinéraires qui quadrillent son espace ", Berryman propose que les lieux de l'Homme soient un des socles de la culture (2005, p. 68), car celle-ci s'incarne dans des lieux. Il précise que "pour l'enfant, l'objet, dans une perspective d'éco-ontogenèse, est d'explorer concrètement l'environnement et la nature et de se sentir chez lui dans le monde [...]. Pour le petit enfant, une approche privilégiée correspond à encourager l'exploration via des histoires et des gestes qui permettent de soutenir ces petites explorations et de faire ressentir à l'enfant une empathie face à la nature et aux animaux » (Berryman, 2003, p. 218). L'ensemble des travaux se référant à ce courant souligne ainsi l'une des dimensions fondamentales de l'ERE, « soit la prise en compte du rapport à la nature et à l'environnement dans le développement humain, dans l'ontogenèse humaine» (Berryman 2003, p. 224). En prolongeant sa réflexion, cet auteur propose de centrer l'ERE sur «les lieux et la communauté » (Berryman, 2005).

19 Ainsi, la notion de lieu, utilisée par Pruneau d'une part et d'autre part par Berryman en référence à l'écoformation, comprend de fortes composantes expérientielles et affectives de l'être au monde et de son monde vécu, et renvoie directement aux conceptions développées dans le courant de la géographie sociale selon lesquelles l'appropriation du lieu ne peut se construire que par l'intégration progressive d'un 
sentiment local partagé, à travers un processus de réappropriation des savoirs locaux et donc d'apprentissages collectifs. Les composantes expérientielles et affectives de l'être au monde concourent alors dans ce cadre à la formation d'une identité territoriale selon les processus décrits dans la géographie sociale.

L'autobiographie environnementale est définie par Bachelart (2009a, p. 125) comme une voie d'" accès à ce fond expérientiel qui nous relie à des milieux » (2009b, p. 50) et comme "une méthodologie de recherche et de formation issue des «approches biographiques» qui ont également exploré les concepts "d'identité écologique » (Thomashow, 1995), de " sensibilité environnementale " ${ }^{8}$ et "d'attachement aux lieux " (Chawla, 2002 $)$. Ces divers travaux sur l'autobiographie environnementale tendent à mettre à nu «la manière dont l'individu se développe en relation à ses environnements" et ce qui "génère ou freine les comportements proenvironnementaux" (Bachelart, 2009a, p. 125). Elle affirme que "les récits produits s'ouvrent à une éthique du care qui intègre la vulnérabilité et la dépendance à autrui » (Bachelart, 2009b, p. 51).

21 Ces trois courants soulignent ainsi l'importance de la prise en compte, dans l'ERE, du rapport à l'environnement pris au sens de territoire, lieu de vie. Nous retrouvons cette préoccupation majeure dans des mouvements d'éducation populaire, comme les CEMEA pour lesquels le développement de l'enfant reste au cœur des préoccupations éducatives :

Cette idée forte de l'éducation nouvelle sur la valeur de l'enfance revêt une grande importance en matière d'EE. Il convient donc de mettre en œuvre des activités de découverte qui intègrent aussi le ludique, le sensible et qui laissent de la place à l'activité de l'enfant. Après tout, un arbre c'est aussi un élément à grimper! Une rivière est aussi un espace de jeux avec des barrages et des petits bateaux! (CEMEA, 2016) ${ }^{10}$

Il en est de même au sein du mouvement scout, pour qui le positionnement du rapport à la nature semble aujourd'hui très présent : « La vie quotidienne en pleine nature [...] fournit [...] les moyens d'une éducation basée sur des rapports harmonieux entre l'homme et son milieu de vie, assez loin des problèmes de consommation et de confort $»^{11}$. Dans ce cadre, et en phase avec le courant de l'éco-ontogenèse, les jeunes sont immergés dans le milieu, en tant que milieu de vie et en tant que milieu éducatif avec l'idée, conformément à l'épistémologie de la géographie sociale, que le territoire est un « espace informé » qui construit une compréhension du monde et une identité.

Ces approches à fortes consonances expérientielles et affectives se différencient donc des courants d'ERE plus positivistes, basés en France sur des études de milieux que nous avons déjà décrites (Girault et Debart, 2006) comme les activités d'éveil (tiers temps pédagogique institué en France en 1969 à l'école élémentaire) basées sur des études pluridisciplinaires supposées portées sur des sujets correspondant aux intérêts de l'enfant et réalisées dans son milieu de vie (Giolitto, 1982). Il s'agissait de "faire connaître l'environnement et l'utiliser comme facteur de motivation et instrument de formation » (Giolitto, 1982). Le tiers temps pédagogique constituait également un cadre intégrateur pour les classes transplantées, dispositifs conçus comme « des classes de vie où le milieu est le centre privilégié de toutes les activités " (Circulaire du 6 mai 1971, citée par Giolitto, 1982). Les classes transplantées permettent aux élèves de découvrir d'autres milieux de vie, de se rapprocher de la nature et parfois d'en avoir une première vraie découverte, etc. Les classes transplantées prendront le nom de «classes de découverte » en 1982, «classes patrimoine » et «classes culturelles » en 1988, puis 
«classes environnement » en 1993. En 1997, le réseau École et Nature indiquait que les classes d'environnement constituaient « l'un des dispositifs les plus opérationnels (et les plus utilisés) pour faire de l'éducation à l'environnement ».

Enfin, il nous faut aussi faire référence à l'approche interprétative de l'ERE telle que décrite par Robottom et Hart (1993) et qui est centrée sur les rapports entre l'apprenant et l'environnement: d'une part, rapport affectif, symbolique (en convergence avec l'éco ontogenèse et l'écoformation) et d'autre part, rapport cognitif (en convergence avec des pratiques éducatives plus scientifiques, rationnelles et positivistes), ce qui renvoie encore une fois à l'espace symbolique de la géographie sociale (Debarbieux, 2009). L'objectif est de développer un rapport étroit et une compréhension de l'environnement dans une perspective d'empathie. La démarche éducative repose sur l'interaction et le contact direct entre l'apprenant et son territoire perçu comme lieu de vie.

\section{Courants d'éducation relative à l'environnement se focalisant sur le territoire en tant que « lieux de pouvoirs "}

Les courants de recherche en ERE qui s'ancrent le plus dans des problématiques liant territoire et pouvoir sont sans aucun doute ceux de la critique sociale définie par Robottom et Hart (1993) et ceux qui sont centrés sur la communauté, étant entendus que ces deux courants sont largement perméables entre eux. Ils partagent tout d'abord trois principes fondamentaux: l'accountability (responsabilité), le commitment (engagement) et l'empowerment. Le terme d'empowerment est de nos jours très largement utilisé tant par des mouvements activistes, des partis politiques, des ONG... et ce dans des sens parfois très différents. Les sources d'inspirations de la notion d' empowerment semblent multiples et peuvent être retracées «dans des domaines aussi variés que le féminisme, le freudisme, la théologie, le mouvement black power ou le gandhisme » (Simon 1994 et Cornwall, Brock 2005, cités dans Calvez 2009, p. 736). Dès l'émergence de ce concept, l'empowerment a renvoyé à des principes, tels que la capacité des individus et des collectivités à agir pour assurer leur bien-être ou leur droit de participer aux décisions les concernant, qui guident la recherche et l'intervention sociale auprès des populations marginalisées et pauvres depuis plusieurs décennies aux États-Unis (Simon, 1994). Selon Baillon (1993, p. 163), ces trois courants sont également ancrés dans le territoire des apprenants, à partir de l'hypothèse selon laquelle les projets les plus porteurs seraient ceux qui se centrent sur l'élève et son cadre de vie. C'est donc dans le concret que le jeune peut découvrir ce que recouvre la notion de " citoyenneté sociale ». Dans ce cadre, le territoire est perçu comme lieux de pouvoir et s'inscrit le plus souvent également dans l'éducation à la citoyenneté. La dimension locale peut alors être perçue comme un principe fondateur qui favorise "l'autonomie des établissements et,ou des autorités locales pour adapter les curriculums et autres programmes établis par les autorités centrales, aux contextes locaux » (Audigier, 2006, p. 42).

Le mouvement de la critique sociale vise donc le développement d'un engagement dans l'action, individuellement et collectivement, afin d'améliorer l'environnement physique et social. Il se rapproche ainsi de l'idée que porte la géographie culturelle selon laquelle c'est la spécificité des représentations des groupes humains qui facilite la compréhension du monde et de sa capacité d'action. L'approche de la critique sociale 
repose principalement sur des processus d'investigation des réalités environnementales, sociales et éducationnelles qui posent problème dans le but de transformer ces dernières. Plus précisément (Robottom et Hart, 1993) rapportent des expériences montrant que ces investigations et cet engagement dans l'action sont plus efficaces quand ils sont collectifs plutôt qu'individuels et en relation avec le milieu de vie des apprenants. Ainsi Hart, en 1992, décrivait plusieurs projets menés dans différents pays notamment au Royaume-Uni où, dans les années 1970 et 1980, se sont multipliés les projets communautaires menés à l'école primaire. En participant à des petits projets réalistes, les élèves et leurs enseignants intégraient les processus de gestion de la communauté et d'aménagement du territoire à un niveau très local. Avec ces exemples, Hart (1992) mettait en avant les leviers favorables à de tels projets : le degré élevé de liberté et d'autonomie des chefs d'établissements des écoles primaires britanniques, la volonté politique d'intégrer les populations locales aux questions d'aménagement, l'engagement d'un personnage clé ayant des responsabilités politiques dans le domaine de l'éducation et ayant favorisé de tels dispositifs et surtout l'aide apportée par des acteurs extérieurs comme les Urban Studies Centres (Centres d'études urbaines), où les élèves et les habitants viennent trouver une aide méthodologique et un accompagnement dans leurs investigations quand ils en ont besoin. En compilant les études menées par les élèves et les habitants, ces centres sont également devenus, selon cet auteur, des lieux de référence et de savoirs co-construits exploités par la communauté dans son ensemble. On peut également citer par exemple, le modèle de recherche-action pour la résolution de problèmes communautaires proposé dès 1988 par Stapp et coll. et qui consiste à engager «les élèves dans un projet collectif de résolution d'un problème social ou environnemental qu'ils ont eux-mêmes repéré dans leur milieu de vie communautaire, et qu'ils mènent en cogestion avec l'enseignant, en alliant l'action à la réflexion critique » (cité dans Sauvé, 1997, p. 234). S'inscrivant dans une démarche similaire ayant pour objet une réflexion sur la participation citoyenne, Girault et coll. (2008) ont souligné d'une part la capacité de jeunes collégiens, habitant en Seine Saint-Denis en France, à s'engager dans une question qui les préoccupe fortement (lecture critique des lieux de vie des élèves, investigations diverses auprès des habitants et des acteurs locaux) et d'autre part, la manière dont ce travail a changé leur regard sur leur territoire et sur leur place dans ce territoire (sentiments d'appartenance et désir d'engagement).

D'autres auteurs ont abordé des stratégies d'ERE à la lumière du concept d' empowerment, et notamment Carine Villemagne (2005) qui a exploré le champ de l'ERE en milieu communautaire à travers son travail de recherche qui renvoie également aux concepts territoriaux de la géographie culturelle. Elle en conclut entre autres que les communautés présentent un contexte favorable au développement d'un processus d' empowerment de leurs membres, en intégrant des préoccupations sociales, culturelles, communautaires et écologiques, mais également des valeurs de solidarité, de coopération, de participation, de responsabilité, etc.

Dans ce cadre, les investigations réalisées par les apprenants impliquent un processus d'analyse critique des valeurs et des intérêts sous-jacents, dont la présence caractérise la nature conflictuelle des questions environnementales. Le courant de la critique sociale inscrit donc le rapport à l'environnement dans le domaine de la critique et du changement social. Dans ce courant où l'éducation sociale est particulièrement mise en 
avant, « l'ERE tend à favoriser la coopération dans l'apprentissage et dans l'exercice de l'action environnementale » (Sauvé, 1997, p. 105).

Plus généralement, on aborde ici certaines théories sociales de l'éducation dans lesquelles «l'éducation doit jouer un rôle important dans la reconstruction de la société » (Bertrand, 1998, p. 166) et notamment celle de la pédagogie de la libération développée par Freire (1970), et par extension, dans la géographie culturelle, celle des groupes culturels spécifiques bien individualisés qui «prennent pouvoir» sur leur espace.

\section{Courants de l'éducation relative à l'environnement se focalisant sur le territoire perçu en tant que rapports d'appropriation entre une société et un espace géographique}

Comme nous l'avons posé précédemment à propos des fondements épistémologiques des concepts de "rapport aux territoires » et de "construction sociale du territoire ", les rapports d'appropriation d'une société à son espace géographique génèrent une ambiguïté, selon que l'on se réfère à l'identité du territoire ou à ses ressources. Si l'identité du territoire, qui se réfère davantage au territoire lieu de vie, se retrouve dans divers courants d'interprétation, la référence aux ressources de ce territoire, renvoie plus spécifiquement à l'émergence des courants de l'éducation au développement durable (EDD). En effet, il est aujourd'hui considéré par certains auteurs que «la mise en œuvre du développement durable dans les territoires est un élément clé pour l'engagement de la société vers une croissance revue à la lumière du Grenelle de l'environnement » (Bovar O., 2010). Le lien entre développement durable et territoires est fréquemment repris et formalisé par les chercheurs en sciences humaines toutes disciplines confondues (Zuindeau et Vivien, 2003 ; Lazzeri et Moustier, 2008), et il existe différents types de travaux du côté de l'EDD, dont nous allons dresser les principaux traits.

31 De nombreux travaux font état de l'accompagnement, dans l'éducation formelle ou informelle, des politiques publiques de développement durable ancrées dans les territoires, comme les Agendas 21 au niveau des collectivités territoriales. Dans ces travaux, ce qui est en jeu avant tout est l'exemplarité de ce qui est mis en place, considéré comme reproductible (Legardez, 2011; Lebatteux et Lange, 2013). Il s'agit donc de démarches performatives, lesquelles sont accompagnées d'une réflexion méthodologique ou didactique sur les pratiques éducatives observées et leurs améliorations possibles. Lebatteux (2011) parlera par exemple d'identifier les obstacles à la mise en œuvre de dispositifs. Dans ces travaux cependant, il est fait peu de place aux réflexions épistémologiques concernant l'EDD elle-même, ou encore la façon dont s'opère l'ancrage dans le territoire, même si le développement durable est le cadre privilégié des politiques de développement et des politiques éducatives dans les établissements scolaires.

D'autres travaux (Leininger-Frézal, 2009) se réfèrent à l'ancrage territorial de l'éducation au développement durable, auquel cas il s'agit d'analyser les jeux d'acteurs et de voir leur spécificité et leur évolution par le prisme de l'EDD. "Penser les territoires de l'éducation au développement durable, c'est donc localiser, mais aussi comprendre le rapport des parties prenantes au territoire" Leininger-Frézal (2009 p. 78). Pour cette auteure, les parties prenantes désignent l'ensemble des acteurs 
individuels ou collectifs qui interviennent dans le champ de l'éducation au développement durable. Ce qui est mis en avant, c'est l'interconnexion des acteurs, la nature et la qualité des liens entre les parties prenantes. Ces travaux se posent comme un construit d'indicateurs et de clés de lecture des dispositifs qui sont mis en place. Dans ce cadre, apparaissent progressivement des relations de pouvoirs, des fragilités et des inégalités.

Les travaux les plus nombreux (Adomßent et coll., 2007 ; Larsen 2009 ; Dussault 2010 ; Sterling et Scott, 2011 ; Barthes et Alpe, 2012 ; Barthes, Champollion, 2012) se réfèrent à la modification des formes éducatives face à la prise en compte plus grande des spécificités locales. «La volonté de faire prendre en charge par l'éducation formelle et non formelle de nouvelles questions a généré de nouvelles modalités scolaires, souvent désignées sous le vocable des "éducations à ", émergentes depuis une vingtaine d'années comme l'éducation au développement durable ou l'éducation au patrimoine. Elles ne répondent pas au modèle académique des disciplines scolaires et peuvent remettre en cause la forme scolaire dominante " (Barthes et Alpe, 2012, p. 198). Dans ces travaux, il est fait référence implicitement aux territoires pris comme ressource, ce qui impacte largement les valeurs et la finalité des enseignements qui sont dispensés. Barthes et Maximin (2014) posent le problème en ces termes: "L'éducation au développement durable est-elle pourvoyeuse de savoirs et/ou au service du développement des territoires? » La question de la légitimité des dispositifs mis en place est posée : «Les modes de légitimation des contenus de ces " éducations à ", liés à la fois aux questions traitées et au statut des intervenants posent de nombreux problèmes, en particulier du fait de leur grande faiblesse épistémologique » (Barthes et Alpe, 2012, p. 204 ).

Dans la même lignée des travaux portant sur les relations entre les territoires et la sphère éducative, d'autres se réfèrent à des territoires bien identifiés : ce sont par exemple des villes ou des espaces ruraux, des parcs naturels ou encore des zones urbaines sensibles. Au sein de ces espaces s'élaborent des projets éducatifs qui ne sont pas véritablement liés aux besoins d'apprentissages des publics, mais qui sont directement liés aux territoires qui les portent. De fait, les caractéristiques des territoires concernés jouent fortement sur les projets éducatifs d'écoles. Il est alors considéré que «le territoire devient un acteur des contenus éducatifs à part entière considérant par exemple un projet de développement rural et ses spécificités " (Barthes et Champollion, 2012, p. 49). Dans le même esprit, Larsen (2009) développe le concept de "ville apprenante», puis Dussault (2010) celle de "ville éducatrice " à propos de projets mis en place par la ville d'Angers dans les écoles. On retrouve cette posture dans les travaux internationaux qui portent sur les partenariats avec les universités (Adomßent et coll., 2007 ; Sterling et Scott, 2008).

Enfin, il convient de noter qu'il existe des travaux sur les territoires qui se préoccupent de la relation au temps, en particulier de la conception de leur futur. Le territoire est ici entendu dans une acception géographique du terme. Il ne s'agit pas seulement de l'espace approprié, il s'agit aussi de l'espace dans lequel un individu ou un groupe d'individus se projette. Cette posture renvoie au développement durable et à l'attention particulière qui est portée aux générations futures. Vergnolle Mainar C., Julien M.-P., Léna J.-Y. et Calvet A. (2013) tentent alors de concevoir le futur d'un territoire dans une perspective d'éducation au développement durable. Il s'agit donc autant d'une 
réflexion didactique que d'un positionnement théorique de l'EDD dans sa dimension spatiale (le territoire) et sa dimension temporelle (ici le futur).

\section{Conclusion}

Nous avons montré, dans le cadre de cet article, que les divers courants de recherche et de pratiques associés à l'éducation au territoire revêtent des objectifs et des valeurs différentes, voire même parfois antagonistes. Depuis les années 2000, de nombreuses structures d'accueil des publics au sein d'aires protégées (réserves de biosphère, parcs nationaux, parcs naturels régionales, géoparcs, etc.) développent des activités d'éducation relative à l'environnement centrées sur l'interprétation, l'éducation aux territoires. Si dans certains cas, ces dernières peuvent être associées à l'un ou l'autre des courants que nous avons analysés ci-dessus, d'autres privilégient des approches différentes. Nombreux sont en effet les responsables de ces structures qui se donnent comme objectif de leur politique culturelle, y compris à l'aide de géosymboles, de tendre vers la co-construction du territoire pour que les habitants puissent l'incarner et en faire une référence identitaire; ou encore, ils privilégient une approche plus développementaliste par la promotion du tourisme de nature ou au sein des géoparcs, du géotourisme, qui nécessite la création de structures d'accueil (musées, centres d'interprétation, sentiers d'interprétation, géoroutes, etc.).

Il nous semble donc indispensable de compléter cette réflexion par une analyse des enjeux (axiologiques et ontologiques) et des intérêts des acteurs de la gestion territoriale. Quelles en sont donc les implications économiques, identitaires, patrimoniales, politiques, sociales et autres? Le développement d'une identité territoriale est-il une nouvelle orthodoxie pour l'aménagement du territoire, pour l'implication des acteurs locaux, pour la mise en place d'une «bonne » gouvernance ? Il s'avèrerait enfin intéressant d'analyser les représentations de la nature de même que les usages du territoire au regard de leurs interactions avec les formes bureaucratiques de gestion/conservation qui sont, dans le cas de ces aires protégées, extrêmement diversifiées.

Pour notre part, et dans le cadre d'une visée stratégique liée à un programme de recherche sur les géoparcs, nous allons orienter nos travaux sur des approches diachroniques et comparatives entre divers pays et systèmes éducatifs, pour cerner les rôles, les possibilités, les ressources (musées, sentiers d'interprétation, médias, etc.), les enjeux et les voies de développement des différents géoparcs en matière d'éducation relative à l'environnement, et plus spécifiquement en ce qui a trait à l'éducation au territoire. 


\section{BIBLIOGRAPHIE}

Adomßent, M., Godeman, J. et Michelsen, G. (2007). Transferability of approaches to sustainable developement at universities as a challenge. International Journal of Sustainability in Higher Éducation, 8(4), 385-402.

Andreux, C. (2005). Écoformation et éducation relative à l'environnement montagnard : Entre territoire, nature et culture. Éducation relative à l'environnement : Regards - Recherches - Réflexions, 5, 33-50.

Andreux C. (2001-2002). Éducation à l'environnement en milieu scolaire et partenariat avec les collectivités territoriales. Une expérience en Auvergne ? Éducation relative à l'environnement : Regards - Recherches - Réflexions, 3, 155-166.

Andreux, C. et Brault, C. (2003). Paysages en Combrailles - Guide recueil et pédagogique. ClermontFerrand: CRDP d'Auvergne.

Ardrey, R. (1966). The territorial imperative. New York : Atheneum Press.

Audigier, F. (2006). Évaluer les effets de l'éducation sur la citoyenneté. Dans Direction générale de l'Enseignement scolaire (dir.), Actes du séminaire national « La citoyenneté par l'éducation » (p 37-58). Paris : ministère de l'Éducation nationale.

Bachelart, D. (2009a). Autobiographie environnementale : explicitation et exploration de l'expérience formatrice. Dans Catherine Guillaumin, Sébastien Pesce et Noel Denoyel. Pratiques réflexives en formation. Ingéniosité et ingénieries émergentes (p. 125-154). Paris : L'Harmattan.

Bachelart, D. (2009b). Anthropologie du sensible : apport de l'éthique de la sollicitude à l'égard du monde « non-humain ». Éducation relative à l'environnement : Regards - Recherches - Réflexions, 8, 3555.

Baillon, R. (1993). Le lycée, une cité à construire. Paris : Hachette.

Barthes, A., Blanc-Maximin S., Alpe, Y. et Floro, M. (2014). L'éducation au patrimoine : pourvoyeuse de savoirs et/ou au service des territoires? Colloque international « Les éducations à, un levier de transformation du système éducatif »,17-19 novembre, Rouen (actes en ligne : https:// halshs.archives-ouvertes.fr/halshs-01183403).

Barthes, A. et Alpe, Y. (2012). Les « éducations à », un changement de logique éducative? L'exemple de l'éducation au développement durable à l'université. Spirale 50, « Les éducations à... : nouvelles recherches, nouveaux questionnements ? ", 197-209.

Barthes, A., et Champollion, P. (2012). Éducation au développement durable et territoires, Évolution des problématiques, modification des logiques éducatives, et spécificité des contextes ruraux. Éducation relative à l'environnement : Regards - Recherches - Réflexions, 10, 36-51.

Berryman, T. (2003). L'éco-ontogenèse : les relations à l'environnement dans le développement humain - d'autres rapports au monde pour d'autres développements. Éducation relative à l'environnement : Regards - Recherches - Réflexions, 4, 207-228.

Berryman, (2005). Réapprendre à habiter ici et entre nous : une éducation centrée sur les lieux et la communauté. Éducation relative à l'environnement : Regards - Recherches - Réflexions, 5, 65-86.

Berryman, T. (2007). L'autobiographie environnementale : la prise en compte des dimensions écologiques dans les histoires de vie. Dans, Recueil des communications - Colloque international : 
Le biographique, la réflexivité et les temporalités : Articuler langues, cultures et formation. [331-334]. Université François-Rabelais, Tours, 25-27 juin 2007.

Bretherton, C. (2001). Ecocentric Identity and Transformation Politics. International Journal of Peace Studies, 6(2). Récupéré de : www.gmu.edu/programs/icar/ijps.

Bertrand, Y. (1998). Théories contemporaines de l'éducation. Montréal : Éditions Nouvelles.

Bertacchini, Y. (2008). L'environnement et la construction de la réalité : Introduction à l'intelligence territoriale. Paris : les Cahiers de la SFSIC, $n^{\circ} 2$.

Buttmann, G. et Ratzel, F. (1977). Leben und Werk einer deutschen Geographen. Stuttgart: Wissenschaftliche Verlagsgesellsschaft.

Bobek, H. (1969). Sozialgeographie - Räumliche Strukturen der Gesellschaft. Deutscher Geographentag Bad Godesberg, 1967, Tagungsbericht und Wiss. Abhandlungen, Wiesbaden, 1969, 199-207.

Bonnemaison, (1981). Voyage autour du territoire. L'espace géographique, 4, 249-262.

Bonnemaison, J. (1996). Gens de pirogue et gens de la terre. Paris : Éditions de l'ORSTOM.

Bonnemaison, J. (2000). La géographie culturelle. Paris : Éditions du CTHS.

Bovar, O. (2010). Des indicateurs du développement durable pour les territoires. Revue $d u$ Commissariat général au développement durable, Service de l'observation et des statistiques, La documentation française.

Calvès, A-E. (2009). « Empowerment » : généalogie d'un concept clé du discours contemporain sur le développement. Revue Tiers Monde 4/2009 ( $\left.n^{\circ} 200\right)$, p. 735-749 - Accès en ligne : www.cairn.info/revue-tiers-monde-2009-4-page-735.htm.

CEMEA (2016). Consultation du site en février 2016 : www.cemea.asso.fr/spip.php ?article951.

Chawla, L. (2002). Growing up in an urbanizing world. Londres : Earthscan.

Claval, P. (1973). Principes de géographie sociale. Paris : Éditions M.Th. Guénin, Librairies techniques.

Claval, P. (1995). Géographie culturelle. Paris : Nathan, Collection « Fac Géographie ».

Cornwall, A. et Brock, K. (2005). Beyond Buzzwords « Poverty Reduction », « Participation » and «Empowerment » in Development Policy. United Nations Research Institute for Social Development (UNRISD).

Cottereau D. (2000). Évaluer la mise en forme du lien éco-logique. Éducation relative à l'environnement : Regards - Recherches - Réflexions, 2, 173-181.

Cottereau, D. (2001). Formation entre terre et mer. Paris : L'Harmattan.

Debarbieux, B. (2009). Territoire-Territorialité-Territorialisation : aujourd'hui encore, et bien moins que demain. Dans Martin Vanier (dir), Territoires, Territorialité, Territorialisation. Controverses et perspectives (p.75-89). Rennes : Presse universitaire de Rennes.

Debardieux B. (1999). Le territoire : Histoires en deux langues. Dans Christine Chivallon, Pascal Ragouet et Michael Samers, Discours scientifique et contextes culturels. Géographies françaises à l'épreuve postmoderne (p. 33-34). Bordeaux: Maison des Sciences de l'homme d'Aquitaine.

Delarge, A. (2001). Pratiques interprétatives en muséologie. Études de communication, 24, 57-70.

Di Méo, G. (1994). Patrimoine et territoire, une parenté conceptuelle. Espaces et Sociétés, 78, 15-34.

Di Méo, G. (1997). Les territoires du quotidien. Paris : L'Harmattan. 
Di Méo, G. (1998). Géographie sociale et territoires. Paris : Nathan.

Di Méo, G. et Buléon, P. (2005). L'espace social. Lecture géographique des sociétés. Paris : Armand Colin Dussaux, M. (2010). L'éducation au développement durable, nouvelle politique éducative locale? Dans Marie-Christine Zelem, Odile Blanchard et Didier Lecomte (dir.), L'éducation au développement durable: De l'école au campus (p. 123-139). Paris : L'Harmattan.

Fortin-Debart C. et Girault Y. (2009). De l'analyse des pratiques de participation citoyenne à des propositions pour une Éducation à l'environnement. Éducation Relative à l'Environnement : Regards Recherches - Réflexions, 8, 129-145.

Freire, P. (1970). Pedagogy of the oppressed. New York: Herder and Herder.

François, L (dir.) (2008). Intelligence territoriale. Cachan : Lavoisier.

Frémont, A., Herin R., Chevalier J. et Renard J. (1984). Géographie sociale. Paris : Masson.

George, P. (1945). Géographie sociale du monde. Paris : Presses universitaires de France, Collection

«Que sais-je?, n 197.

Giolitto, P. (1982). Pédagogie de l'environnement, Paris : Presses universitaires de France.

Girault, Y., Auzou E. et Fortin Debart, C. (2008). De la lecture critique du territoire à la notion d'empowerment : étude de cas d'un projet mené avec des adolescents en banlieue parisienne. Éducation relative à l'environnement : Regards - Recherches - Réflexions, 7, 175-197.

Girault, Y. et Debart, C. (2006). État des lieux des perspectives en matière d'Éducation relative à l'environnement à l'échelle nationale. Rapport de recherche. Paris : Muséum national d'Histoire naturelle USM Muséologie et médiation des sciences. Consultable sur le site Espace ressources : www.espace-ressources.uqam.ca.

Gravel, H. et Pruneau, D. (2004). Une étude de la réceptivité de l'environnement chez les adolescents. Revue de l'Université de Moncton, 35(1), 165-167.

Hay, R.B. (1988). Toward a theory of sense of place. Journal of Ecosophy, 5(4), 159-164.

Hummon, D.M. (1986). City mouse, country mouse: The persistence of community identity. Qualitative Sociology, 9(1), 3-25.

Hart, P. (1992). Children's participation. From tokenism to citizenship. Florence: UNICEF (United Nations Children's Fund), Innocenti Research Centre.

Hartke, W. (1969). Gedanken ueber die Bestimmung von Raeumen gleichen sozialgeographischen Verhaltens. Erdkunde, 13, 426-436.

Howard, E. (1920). Territory in bird's life. New York: Murray.

Houssay-Holzschuch, M. (2006). Antimonde. Géographies sociales de l'invisible - introduction du numéro spécial. Géographie et Cultures, 57, 3-8.

Jickling, B. (1992). Why I don't want my children to be educated for sustainable development. The journal of environmental education, 23(4), 5-8.

Landel, PA. (2006). Invention de patrimoines et construction des territoires. Dans Gumuchian, H. et Pecqueur, B. (dir.), La ressource territoriale (p. 149-157). Paris : Édition L'Harmattan.

Landel, P-A. et Senil N., (2009). Patrimoine et territoire, les nouvelles ressources du développement. Développement durable et territoires. Récupéré de : https://

developpementdurable.revues.org/7563. 
Larsen, K. (2009). La ville apprenante, la nouvelle recette du développement régional.

L'observateur de l'OCDE, 6

Lazzeri Y., Moustier, E. (2008). Le développement durable. Du concept à la mesure. Paris : L'Harmattan.

Lebatteux, N. (2011). Penser global et agir local ? Des obstacles à la mise en œuvre d'un Agenda 21 scolaire. Dans A. Legardez \& L. Simonneaux, Développement durable et autres questions d'actualité. Questions socialement vives dans l'enseignement et la formation (pp. 181-194). Dijon : Éducagri éditions Lebatteux, N. et Lang, J-M. (2013). Des effets de la participation des élèves au pilotage d'Agendas 21 scolaires : entre acquisition de connaissances, compétences et attitudes. 3e colloque international de l'Association pour des Recherches Comparatives en Didactique (ARCD). Savoirs, compétences : Approches comparatives de l'organisation des formes de l'étude. Aix-Marseille Université.

Legardez, A. (2011). Formation et pratiques d'enseignement en questions. Rapport aux savoirs sur le développement durable en contexte scolaire : obstacles à la mise en œuvre d'un Agenda 21 en France, 13, 179-199

Leininger-Frézal, C. (2009). Le développement durable et ses enjeux éducatifs. Acteurs, savoirs et stratégies territoriales. Thèse de doctorat inédite, Géographie. Université Lumière - Lyon II.

Linck, T. (2012). Économie et patrimonialisation. Développement durable et territoires, 3 (3). Récupéré de : https://developpementdurable.revues.org/9506.

Perazzone A. Camino E. Bertolino F. (1995). Institution scolaire et zones protégées du Piémont (Italie) : le cas particulier d'une expérience coordonnée . Aster, n² 29, Paris, INRP, 101-130.

Pineau, G. et Marie-Michèle (1983, réédité 2012). Produire sa vie : autoformation et autobiographie. Paris : Téraèdre.

Pineau, G. (1992). De l'air. Essai sur l'écoformation. Paris / Montréal : Païdeia.

Pineau, G. et Le Grand, J-L. (1992, réédité 2013). Les histoires de vie. Paris : Presses universitaires de France

Pineau, G. (dir.) (2001). Pour une écoformation. Former à et par l'environnement » Éducation permanente, 148.

Piveteau, V. (2010). Territoire-formation-développement : un triptyque à revisiter par temps changeant, Développement des territoires et formation (2). Éducation permanente, 185

Pruneau, D. et Chouinard, O. (1997). Un modèle d'intervention pédagogique qui favorise la relation personne/groupe/social/environnement. Dans Mohamed Hrimech et France Jutras (eds), Défis et enjeux de l'éducation dans une perspective planétaire (p. 107-123). Sherbrooke : Éditions du CRP

Pruneau, D., Chouinard O. et Gravel H. (1998-1999). Le chainon manquant la compréhension de la relation personne, groupe social, environnement. Éducation relative à l'Environnement : Regards Recherches - Réflexions, 1, 209-215.

Pruneau, D., Gravel, H. et Ouattara I., (2002). Les relations que les adolescents entretiennent avec leur environnement. Revue des sciences de l'éducation, 28 (3), 565-585.

Proshansky, H.M., Fabian, A.K. et Kaminoff, R. (1983). Place identity. Journal of Environmental Psychology, 3, 57-83.

Raffestin, C. (1980). Pour une géographie du pouvoir. Paris : Librairie technique (Litec)

Relph, E. (1976). Place and placelessness. London : Pion. 
Ripoll, F. et Veschambre, V. (2002). Face à l'hégémonie du territoire : éléments pour une réflexion critique. Dans Yves Jean et Christian Calenge (dir.), Lire les territoires. Tours : Publications de la MSH, collection « Perspectives Villes et territoires », $n^{\circ} 3,261-288$.

Robottom, I. et Hart, P. (1993). Research in environmental education. Deaking, Australie : Deaking University Press.

Sauvé, L. (1997). Pour une éducation relative à l'environnement - Éléments de design pédagogique, Guide de développement professionnel à l'intention des éducateurs. Montréal : Guérin - Eska, 2e édition

Sauvé, L. (2006). L'organisation et la structuration du secteur de l'éducation en réponse au programme onusien du développement durable. Dans « Former et éduquer pour changer nos modes de vie », Liaison Énergie-Francophonie, 72, 33-41.

Simon, B. (1994). The Empowerment Tradition in American social Work : a History. New York : Columbia University Press.

Stapp, W. B. et Bull, J. (1988). Education in action - A community problem solving program for schools. Dexter (Michigan): Thompson-Shore.

Sterling, S. et Scott, W. (2008). Higher education and ESD in England: a critical commentary on recent initiative. Environmental Education Research, 14(4), 383-504.

Tanner, T. (1980). Significant life experiences: A new research area in environmental education. The Journal of Environmental Education, 11(4), 20-24.

Tilden, F. (1957, réédition 2008). Interpreting our Heritage. University of North Carolina Press Thomashow, M. (1995). Ecological identity: Becoming a reflective environmentalist. Cambridge: MA: MIT Press.

Tuan, Y.F. (1991). Language and the making of place: A narrative descriptive approach. Annals of the Association of American Geographers, 81(4), 684-696.

Vergnolle-Mainar, C., Julien M-P., Léna J-Y. et Calvet A. (2013). La question de la projection vers le(s) futur(s) possible(s) : quels points d'appui ? Penser l'éducation, hors-série décembre, 257-273.

Vergnolle-Mainar, C., Julien, M-P., Chalmeau, R., Léna, J-Y. et Calvet A. (2014). Concevoir le futur d'un territoire dans une perspective d'éducation au développement durable. Vertigo, 14 (1)(Accès en ligne : https://vertigo.revues.org/14690).

Vidal de la Blache, P. (1913). Des caractères distinctifs de la géographie. Chicoutimi : Les classiques des sciences sociales (Accès en ligne : http://classiques.uqac.ca)

Villemagne, C. (2005). L'éducation relative à l'environnement en milieu communautaire urbain. Un modèle théorique en émergence enrichi de l'exploration collaborative de pratiques éducatives. Thèse de doctorat inédite. Université du Québec à Montréal.

Zuindeau, B. ;Vivien F.D. (2003). Territoire et développement durable. Paris : L'Harmattan.

\section{NOTES}

1. Pour une approche linguistique de ce concept: Debardieux B. (1999). Signalons également que le présent article s'appuie entre autres sur des écrits recensés dans le cadre de l'élaboration des Repères théoriques du Centr'ERE : https://centrere.uqam.ca/ wp-content/uploads/sites/12/2014/02/LAFITTE_Bibliographie.pdf 
2. Voir à ce sujet l'article de Alexandre Delarge (2001).

3. Cette expression proposée par Hugues de Varine a été reprise par Georges-Henri Rivière au début des années 50, pour focaliser les présentations muséales sur l'écologie et l'ethnologie régionale. Ce concept a été expérimenté à partir de 1968 dans les parcs naturels régionaux du Mont D'Arrée, Ouessant, Grande lande, Camargue, Mont Lozère. Hugues de Varinne "politise plus le concept ", à partir d'aspirations participatives et autogestionnaires. Il contribue à créer avec Marcel Évrard, l'Écomusée du Creuzot (1971) qui est devenu depuis lors, un phénomène culturel d'ampleur internationale.

4. S'il nous paraissait indispensable de faire référence à ces courants qui ont de nos jours de très nombreux développements (par exemple, la création des sentiers d'interprétation au Canada), il n'est pas possible toutefois d'en présenter ici toute la richesse, car cela nous éloignerait de notre propos.

5. À titre exploratoire, le lecteur pourra se référer au volume 10 de la revue Éducation relative à l'environnement - Regards, Recherches, Réflexions. « Habiter : L'ancrage territorial comme support d'éducation à l'environnement». http://www.revue-ere.uqam.ca/ categories/volumes/v10.html

6. En France, il existe une équipe de chercheurs reconnus internationalement (GREF, Groupe de Recherche surl'Écoformation, Université de Tours) qui travaille spécifiquement sur cette problématique. Ces chercheurs insistent sur la nécessité d'intégrer les principes de l'écoformation dans les actions d'éducation relative à l'environnement. C'est notamment le principe de l'alternance entre pédagogie de projet et pédagogie de l'écoformation par exemple.

7. Dans cet article, Tom Berryman présente notamment les travaux de 5 auteurs qui ont contribué au développement de l'éco-ontogenèse dont Harold Searles (1960/1986).

8. Voir à ce sujet Gravel et Pruneau (2004).

9. Dans sa contribution, Chawla (2002) analyse les conditions environnementales qui sont propices au développement des enfants vivant en milieu urbain.

10. À L'origine de la naissance, en France, des CEMEA, il y a l'histoire d'une époque, celle de 1936 et du Front Populaire, époque pleine de promesses, de projets audacieux, de bouillonnement d'idées neuves et de réformes sociales. [...] C'est le 12 décembre 1938, qu'est déposée la déclaration à la préfecture de police de l'association des Centres d'entraînement pour la formation du personnel des colonies de vacances et des Maisons de campagne des écoliers. [...]. En 1943 l'association prendra le nom de Centre d'entraînement aux méthodes de pédagogie active. Elle proposera le premier stage d'Instructeurs à Marly-le-Roi. Consultation du site en février 2016

11. Extrait du « Projet éducatif des Éclaireuses Éclaireurs de France ». Consultation du site en mai 2015: http://www.eedf.fr/ressources/downloads/ le_projet_educatif_des_eedf.pdf 


\section{RÉSUMÉS}

La géographie développe une réflexion épistémologique sur les sociétés et leurs territoires et précise, à travers les courants de la géographie sociale et culturelle, les liens entre les savoirs et leurs territoires. Nous examinons ces divers ancrages épistémologiques, lesquels sont largement repris dans les réflexions sur les modalités, valeurs et finalités des éducations relatives à l'environnement et au développement durable. Cela permet de proposer une typologie des approches théoriques portant sur la question émergente de l'éducation aux territoires.

Geography develops an epistemological reflection on society and their territories. Through the currents of social and cultural geography, the links between knowledge and their territories are scrutinized. This article examines these various epistemological anchors, which are widely represented in reflections on methods, values and goals of education for environment and sustainable development. This analysis enables us to propose a typology of theoretical approaches on the emerging issue of territorial education.

\section{INDEX}

Mots-clés : épistémologie, géographie, éducation relative à l'environnement, éducation au développement durable, éducation aux territoires

Keywords : epistemology, geography, environmental education, education for sustainable development, territorial education

\section{AUTEURS}

\section{YVES GIRAULT}

Yves Girault est Professeur au Muséum national d'Histoire naturelle de Paris, UMR Patrimoines locaux et gouvernance. Il est également chercheur régulier au centre de recherche en éducation et formation relatives à l'environnement et à l'écocitoyenneté (UQAM, Montréal). Ses travaux portent notamment sur l'éducation à l'environnement/ à l'écocitoyenneté dans le contexte muséal. Il est (2015/2018) responsable d'un projet de recherche Européen H2020-MSCA-RISE-2014 (Excellence Science) qui analyse notamment les processus de patrimonialisation (nature, culture) dans un contexte de labélisation internationale (UNESCO).

\section{ANGELA BARTHES}

Angela Barthes est Professeure à l'Université d'Aix-Marseille, membre du laboratoire Apprentissage, Didactique, Évaluation, Formation (ADEF) et membre associée à l'UMR Patrimoines locaux et gouvernance (MNHN Paris). Ses recherches concernent la transmission des savoirs et les éducations dans le développement des territoires (éducation au développement durable, éducation au patrimoine, transmission des savoirs dans le développement rural (aires naturelles protégées, patrimoines, développement local). Elle participe actuellement au projet de recherche Européen H2020-MSCA-RISE-2014 (Excellence Science). 